\title{
Practice variation and trends in the management of incomplete and missed spontaneous abortion: Informing a multisite quality improvement project.
}

\section{Megg Wylie, Amelia Srajer, Kevin Lonergan, Philippa Brain, Eddy Lang}

Background: Practice variation in the management of spontaneous abortion exists in the Emergency Department (ED). We developed a multisite retrospective study to assess how the management of spontaneous abortion has changed over 2014-2019 across four Calgary EDs, with emphasis on assessing variation and trends between non-operative (medical/expectant) and operative (dilatation and curettage) management. Medical management has been increasingly indicated as effective, yet a knowledge gap exists regarding its use. Knowledge of that proportion and physician-level practice variation will facilitate educational and audit and feedback style initiatives. Results provide justification and supporting data for said initiatives, which may be extrapolated to elsewhere.

Implementation: Two medical students are heading the day to day work of this project, with support from a principal investigator with the Department of Emergency Medicine in Calgary. We also have the support of a data manager and the head of pregnancy loss in the region. This study was implemented as a quality improvement project. Therefore, the Conjoint Health Research Ethics Board at the University of Calgary was consulted to ensure the project qualified as a quality improvement and that our privacy protections were appropriate. With approval from the ethics board, we needed the data to analyze and assess. To do so, we utilized Sunrise Clinical Manager (SCM) to retrospectively collect data. Sunrise Clinical Manager, a system utilized in Calgary EDs to track patient and department information, was accessed to collect administrative data. Sustaining this work will involve the continued efforts of the described team, largely in writing up the results and disseminating them via audit and feedback procedures.

Evaluation Methods: Using SCM, data were retrospectively collected for patients coded with International Classification of Disease (ICD-10) codes O03.4 (incomplete spontaneous abortion without complication) or O02.1 (missed abortion) who presented to an ED in Calgary (Foothills Medical Centre, Peter Lougheed Centre, South Calgary Hospital, and Rockyview General Hospital) over 2014-2019. We collected patient and environmental factors to allow for the examination of unintended associations. Hemoglobin, HCG level, CTAS code, PIA (time to $\mathrm{MD}$ ), and U/S result (to confirm diagnosis) were collected. Variables regarding length of stay, procedures received (D\&Cs, or other), and returns to care (within 72 hours, and 7 days) were collected. Return to care for future $\mathrm{D} \& \mathrm{C}$ was considered a proxy for failed non-operative management. Demographic and practice data were collected on ED physicians who saw a minimum of 15 patients from our cohort, to gain understanding of trends in practice. Data were analyzed using Chi-square and Mann-Whitney U tests. 
Results: Within our cohort, 1110 (28.9\%) patients received a D\&C. The remaining 2735 (71.1\%) patients were managed non-operatively. Variation and trends were present between sites, with rates of D\&Cs ranging from $15.8 \%$ to $33.5 \%(\mathrm{p}<0.001)$. The rate of D\&Cs decreased from $34.2 \%$ in 2014 to $22.6 \%$ in 2019 across all sites ( $<<0.001$ ), and $11.6 \%$ absolute and $33.9 \%$ relative reduction; yet there was minimal variation over time in rates of ED returns and returns resulting in D\&Cs. $78.6 \%$ of physicians who saw $\geq 15$ patients were female, with female physicians responding to $81.8 \%$ of our cohort

\section{Advice and Lessons Learned:}

1) Our first suggested lesson is to have a clear plan regarding deadlines and timelines, but to also have room for flexibility. At some times the work on this project was slowed to allow effective collaboration with the obstetrics and gyneocology department, or to allow for the refinement of data management. By setting realistic timelines, team members were encouraged to progress the work in a timely fashion. However, by having flexibility the team was able to adapt to roadblocks along the way.

2) A second lesson would be the importance of meaningful collaboration between departments. Though the setting of the project was within Calgary EDs, the topic and content have clear relevance to obstetics and gynaecology. By consulting with members of the obstetrics and gynaecology department we were able to clarify our objectives and have a better understanding of local contextual factors that influenced our results. 